EPJ Web of Conferences 71, 00117 (2014)

DOI: $10.1051 /$ epjconf / 20147100117

(C) Owned by the authors, published by EDP Sciences, 2014

\title{
Interplay between chromoelectric and chromomagnetic gluons in Yang-Mills thermodynamics
}

\author{
Chihiro Sasaki ${ }^{1, a}$ \\ ${ }^{1}$ Frankfurt Institute for Advanced Studies, D-60438 Frankfurt am Main, Germany
}

\begin{abstract}
We propose an effective theory of SU(3) gluonic matter where interactions between color-electric and color-magnetic gluons are constrained by the center and scale symmetries. Through matching to the dimensionally-reduced magnetic theories, the magnetic gluon condensate qualitatively changes its thermal behavior above the critical temperature. We argue its phenomenological consequences for the thermodynamics, in particular the dynamical breaking of scale invariance.
\end{abstract}

\section{Magnetic confinement}

In Yang-Mills (YM) theories at finite temperature $T$ the magnetic screening mass is dynamically generated as a ultra-soft scale $g^{2} T[1,2]$. Consequently, the magnetic sector remains non-trivial in the high temperature phase and in fact, the spatial string tension is non-vanishing for all temperatures $[3,4]$, indicating certain confining properties. Due to this residual interaction, equations of state $(\mathrm{EoS})$ deviate from their Stefan-Boltzmann limit at high temperature. The interaction measure $I(T) / T^{2} T_{c}^{2}$ obtained from $\mathrm{SU}(3)$ lattice simulations is nearly constant above the deconfinement critical temperature $T_{c}$, in the range $T_{c}<T<5 T_{c}[5,6]$.

Several scenarios have been proposed to explain this non-perturbative nature, see e.g. [7-11]. The major assumption in those models is an effective gluon mass being constant in the intermediate $T$ range. In [12] we propose a different effective theory where the non-trivial behavior of $I(T) / T^{2}$ emerges dynamically from chromomagnetic gluons, without relying on any assumption for the gluon thermal mass.

Using the three-dimensional YM theories [13-16], thermal behavior of the magnetic gluon condensate at high temperature is found as [17]

$$
\langle H\rangle=c_{H}\left(g^{2}(T) T\right)^{4},
$$

with

$$
c_{H}=\frac{6}{\pi} c_{\sigma}^{2} c_{m}^{2}
$$

The constants $c_{\sigma}$ and $c_{m}$ appear in the spatial string tension and in the magnetic gluon mass as

$$
\sqrt{\sigma_{s}(T)}=c_{\sigma} g^{2}(T) T, \quad m_{g}(T)=c_{m} g^{2}(T) T .
$$

\footnotetext{
a e-mail: sasaki@fias.uni-frankfurt.de
} 
For $S U(3)$ YM theory $c_{\sigma}=0.566[5]$ and $c_{m}=0.491$ [18].

The non-vanishing string tension $\sigma_{s}$ may support to consider a system where hadronic states, glueballs, can survive in deconfined phase. The scalar glueballs can be introduced as dilatons associated with the scale symmetry breaking through the potential [19]

$$
V_{\chi}=\frac{B}{4}\left(\frac{\chi}{\chi_{0}}\right)^{4}\left[\ln \left(\frac{\chi}{\chi_{0}}\right)^{4}-1\right],
$$

where $B$ is the bag constant and $\chi_{0}$ is a dimensionful constant. The two parameters, $B$ and $\chi_{0}$, are fixed to reproduce the vacuum energy density $\mathcal{E}=\frac{1}{4} B=0.6 \mathrm{GeV} \mathrm{fm}^{-3}$ and the vacuum glueball mass $M_{\chi}=1.7 \mathrm{GeV}[20,21]$, leading to $B=(0.368 \mathrm{GeV})^{4}$ and $\chi_{0}=0.16 \mathrm{GeV}$.

The confinement-deconfinement phase transition is characterized by the Polyakov loop $\Phi$,

$$
\Phi=\frac{1}{N_{c}} \operatorname{tr} \hat{L}, \quad \hat{L}=\mathcal{P} \exp \left[i \int_{0}^{1 / T} d \tau A_{4}(\tau, \vec{x})\right],
$$

which is is an order parameter of dynamical breaking of $Z\left(N_{c}\right)$ symmetry [22]. The two scalar fields, $\chi$ and $\Phi$, are mixed and their potential should be manifestly invariant under $Z\left(N_{c}\right)$ and scale transformation. For $N_{c}=3$, the most general form is given as [23],

$$
V_{\text {mix }}=\chi^{4}\left(G_{1} \bar{\Phi} \Phi+G_{2}\left(\bar{\Phi}^{3}+\Phi^{3}\right)+G_{3}(\bar{\Phi} \Phi)^{2}+\cdots\right)
$$

with unknown coefficients $G_{i}$. In the following calculation, we take only the first term.

\section{Trace anomaly}

We formulate the model accounting for a mixing between chromoelectric and chromomagnetic gluons as

$$
\Omega=\Omega_{g}+\Omega_{\Phi}+V_{\chi}+V_{\text {mix }}+c_{0} .
$$

The color-electric gluon part $\Omega_{g}$ is given in the presence of a uniform field $A_{0}$ as

$$
\Omega_{g}=2 T \int \frac{d^{3} p}{(2 \pi)^{3}} \ln \left(1+\sum_{n=1}^{8} C_{n} e^{-n p / T}\right),
$$

where the coefficients $C_{n}$ are certain functions of the $S U(3)$ group characters [24]. The Haar measure part is

$$
\Omega_{\Phi}=-a_{0} T \ln \left[1-6 \bar{\Phi} \Phi+4\left(\Phi^{3}+\bar{\Phi}^{3}\right)-3(\bar{\Phi} \Phi)^{2}\right] .
$$

We take the following mixing term,

$$
V_{\text {mix }}=G\left(\frac{\chi}{\chi_{0}}\right)^{4} \bar{\Phi} \Phi .
$$

Requiring that a first-order phase transition appears at $T_{c}=270 \mathrm{MeV}$ as found in the lattice results [5], one finds $a_{0}=(0.184 \mathrm{GeV})^{3}, c_{0}=(0.244 \mathrm{GeV})^{4}$ and $G=(0.206 \mathrm{GeV})^{4}$.

At high temperature, the theory in four dimensions should match the three-dimensional effective theory via dimensional reduction. We postulate the matching condition for the gluon condensate as

$$
\frac{\langle\chi\rangle}{\chi_{0}}=\left(\frac{\langle H\rangle}{H_{0}}\right)^{1 / 4} \text {. }
$$


We use the two-loop running coupling,

$$
g^{-2}(T)=2 b_{0} \ln \frac{T}{\Lambda_{\sigma}}+\frac{b_{1}}{b_{0}} \ln \left(2 \ln \frac{T}{\Lambda_{\sigma}}\right), \quad b_{0}=\frac{11}{16 \pi^{2}}, \quad b_{1}=\frac{51}{128 \pi^{2}},
$$

with $\Lambda_{\sigma}=0.104 T_{c}$ [5]. In this model, the changeover in the temperature dependence of the magnetic condensate appears by construction. In YM theories, however, such behavior should emerge dynamically. A qualitative change of the thermal gluon can be in fact seen in the spatial string tension at $T \sim 2 T_{c}[17]$.

This also affects the EoS. One finds an additional contribution to the interaction measure from $\langle H\rangle$ as

$$
\delta I=-B \frac{\langle H\rangle}{H_{0}}+\left(2 b_{0}+\frac{b_{1}}{b_{0}} \frac{1}{\ln \left(T / \Lambda_{\sigma}\right)}\right) \frac{\langle H\rangle}{g^{4}(T) H_{0}} .
$$

The $I / T^{2} T_{c}^{2}$ is monotonically decreasing even at high temperature when no matching to the 3-dim YM is made. The magnetic contribution generates a $T^{2}$ dependence and this results in a plateau-like behavior emerging in $I / T^{2} T_{c}^{2}$ at moderate temperature, $T / T_{c} \sim 2-4$. This property appears due to the residual chromomagnetic interaction encoded in the dilaton, $\chi^{4} \sim H$. The obtained behavior of $I / T^{2} T_{c}^{2}$ with temperature qualitatively agrees with the latest lattice data [6].

\section{Summary}

We have presented an effective theory of SU(3) YM thermodynamics implementing the major global symmetries, the center and scale symmetries. This naturally allows a mixing between the Polyakov loop and the dilaton field. Consequently, the magnetic confinement is effectively embedded and results in deviations of the EoS from their Stefan-Boltzmann limit at high temperature.

Matching to the 3-dimensional YM theory suggests the gluon condensate increasing with temperature in deconfined phase. We have illustrated that this changeover becomes transparent in the interaction measure $I=\mathcal{E}-3 P$ normalized by $T^{2} T_{c}^{2}$, rather than by $T^{4}$. We have found that the role of the magnetic gluon is alternative to the Hard Thermal Loop contribution.

\section{Acknowledgments}

C. S. acknowledges partial support by the Hessian LOEWE initiative through the Helmholtz International Center for FAIR (HIC for FAIR).

\section{References}

[1] A. D. Linde, Phys. Lett. B 96, 289 (1980).

[2] D. J. Gross, R. D. Pisarski and L. G. Yaffe, Rev. Mod. Phys. 53, 43 (1981).

[3] C. Borgs, Nucl. Phys. B 261, 455 (1985). E. Manousakis and J. Polonyi, Phys. Rev. Lett. 58, 847 (1987).

[4] G. S. Bali, J. Fingberg, U. M. Heller, F. Karsch and K. Schilling, Phys. Rev. Lett. 71, 3059 (1993).

F. Karsch, E. Laermann and M. Lutgemeier, Phys. Lett. B 346, 94 (1995).

[5] G. Boyd, J. Engels, F. Karsch, E. Laermann, C. Legeland, M. Lutgemeier and B. Petersson, Nucl. Phys. B 469, 419 (1996).

[6] S. Borsanyi, G. Endrodi, Z. Fodor, S. D. Katz and K. K. Szabo, JHEP 1207, 056 (2012). 
[7] K. -I. Kondo, Phys. Lett. B 514, 335 (2001). E. Megias, E. Ruiz Arriola and L. L. Salcedo, JHEP 0601, 073 (2006).

[8] P. Castorina, D. E. Miller and H. Satz, Eur. Phys. J. C 71, 1673 (2011).

[9] G. W. Carter, O. Scavenius, I. N. Mishustin and P. J. Ellis, Phys. Rev. C 61, 045206 (2000).

[10] R. D. Pisarski, Prog. Theor. Phys. Suppl. 168, 276 (2007).

[11] A. Dumitru, Y. Guo, Y. Hidaka, C. P. K. Altes and R. D. Pisarski, Phys. Rev. D 86, 105017 (2012).

[12] C. Sasaki, I. Mishustin and K. Redlich, arXiv:1308.3635 [hep-ph].

[13] T. Appelquist and R. D. Pisarski, Phys. Rev. D 23, 2305 (1981).

[14] S. Nadkarni, Phys. Rev. D 27, 917 (1983).

[15] N. P. Landsman, Nucl. Phys. B 322, 498 (1989).

[16] K. Kajantie, M. Laine, K. Rummukainen and M. E. Shaposhnikov, Nucl. Phys. B 458, 90 (1996); Nucl. Phys. B 503, 357 (1997).

[17] N. O. Agasian, Phys. Lett. B 562, 257 (2003).

[18] D. Bieletzki, K. Lessmeier, O. Philipsen and Y. Schroder, JHEP 1205, 058 (2012).

[19] J. Schechter, Phys. Rev. D 21, 3393 (1980).

[20] S. Narison, Nucl. Phys. Proc. Suppl. 54A, 238 (1997).

[21] J. Sexton, A. Vaccarino and D. Weingarten, Phys. Rev. Lett. 75, 4563 (1995).

[22] L. D. McLerran and B. Svetitsky, Phys. Lett. B 98, 195 (1981); Phys. Rev. D 24, 450 (1981).

[23] F. Sannino, Phys. Rev. D 66, 034013 (2002).

[24] C. Sasaki and K. Redlich, Phys. Rev. D 86, 014007 (2012). 